\title{
Microstructure and Compressive Performance of Solidified Waste Resin Concentrate
}

\section{Yukai Chen ${ }^{1}$, Xiaoqian Ning ${ }^{2}$, Canpeng $\mathrm{Li}^{1}{ }^{1}$, Shiqing Huang ${ }^{1^{*}}$ and Liping Zhou}

1 School of Mechanics and Construction Engineering, Jinan University, Guangzhou, 510632, China

${ }^{2}$ Reconnaissance Department, Special Police College of Armed Police Force, Beijing, 102211, China

${ }^{3}$ China Nuclear Power Institute Co., Ltd., Shenzhen 518028, China

\begin{abstract}
Keywords: Solidified Waste; CT-Scan; Compression Test; Microstructure
Abstract: To study the microstructure and mechanical property of cement-solidified waste resin concentrate, and research into the feasibility and effectiveness of solidification of waste resin concentrate, the researchers conducted microscopic CT scan on cement-solidified waste, reconstructed 3D model of resin particle distribution, and performed compression test on samples. The result showed that resin particles in cement-solidified waste resin concentrate are evenly distributed, and the comprehensive strength of the cement-solidified waste, though lowered to some extent after soaking, complies with GB 14569.1-2011 both before and after soaking. Therefore, solidification of waste resin concentrate for radioactive waste disposal is feasible and effective.
\end{abstract}

\section{Introduction}

The development of nuclear power, which has greatly improved the energy structure on a global scale, brings huge economic benefits to the society, contributing to the rapid progress of human society. At the same time, radioactive waste, which comes with nuclear energy, also poses an immeasurable potential threat. Therefore, effective and safe disposal of radioactive waste is a key issue concerning the healthy and sustainable development of the nuclear industry and nuclear energy ${ }^{[1-3]}$.

Generally, ion exchange resin is used to absorb radioactive liquid waste produced by nuclear power plant before solidification for disposal. Common solidification methods include cement solidification ${ }^{[4]}$, pitch solidification ${ }^{[5]}$, plastic solidification ${ }^{[6]}$, glass solidification ${ }^{[7]}$, synroc solidification ${ }^{[8]}$ etc., among which cement solidification, with the advantages of reliable performance, easy operation and lower cost etc., is widely used to treat low and medium radioactive waste including waste resin etc.

In this study, cement-solidified waste is prepared by solidification of waste resin concentrate with cement. Through microscopic CT scan and reconstruction of $3 \mathrm{D}$ model, resin particle distribution in the solidified waste is observed, and compressive strength of the solidified waste is also systematically tested before and after soaking in deionized water. The test result shows that cement-solidified waste resin concentrate meets national standards, proving the feasibility and effectiveness of solidification of waste resin concentrate with cement.

\section{Experimental methodology}

There are 15 samples prepared as per GB 145691-2011 standards (see Figure 1.), and cured for 28 days as per national standards. Samples are in cylindrical shape, $5.0 \mathrm{~cm}$ in diameter and $5.0 \mathrm{~cm}$ in height. With shell removed, samples are randomly assigned into two groups: samples 1-7 are directly measured to identify density and compressive strength without soaking (microscopic CT scan on samples 1-3 before compression test; average density $1.71 \mathrm{~g} / \mathrm{cm} 3$ ); samples $8-15$ are soaked in deionized water, which is changed every day, for 90 days before the compression test; average 
density is $1.81 \mathrm{~g} / \mathrm{cm} 3$. Sample 15 is cut into a $20 \mathrm{~mm}$ cube before high-precision microscopic CT scan.

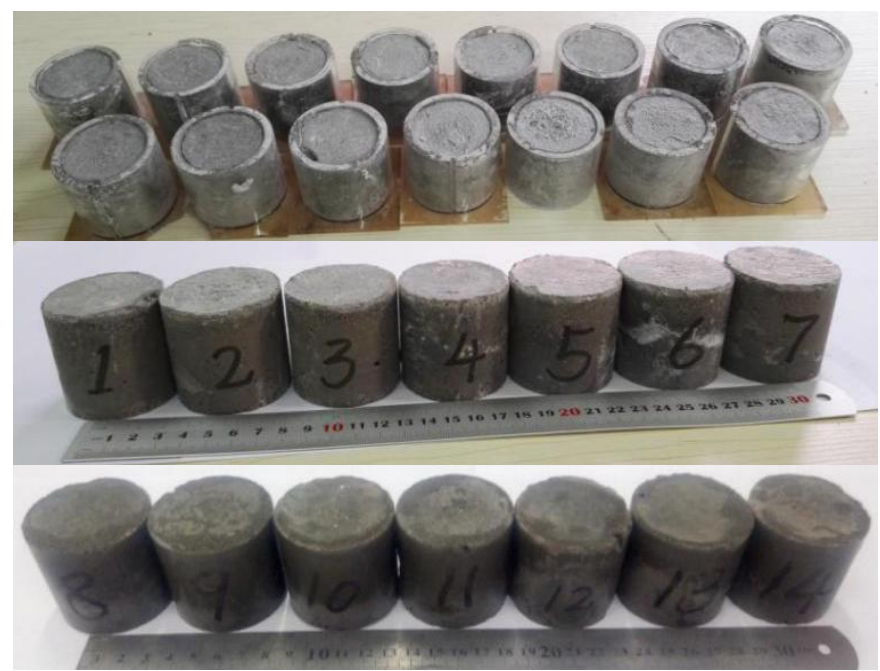

Figure 1. Cement solidified waste samples.

\section{Microscopic CT scan on cement-solidified waste samples}

The samples, containing resin particles sized about $600-700 \mu \mathrm{m}$, need Microscopic CT scan to achieve resolution accuracy. The scanned layer is less than $100 \mu \mathrm{m}$ thick. Overall distribution of resin particles in samples $1-3$ is obtained by microscopic CT scan, $50 \mu \mathrm{m}$ in accuracy, and the scan results of sample 1 are shown in Figure 2.
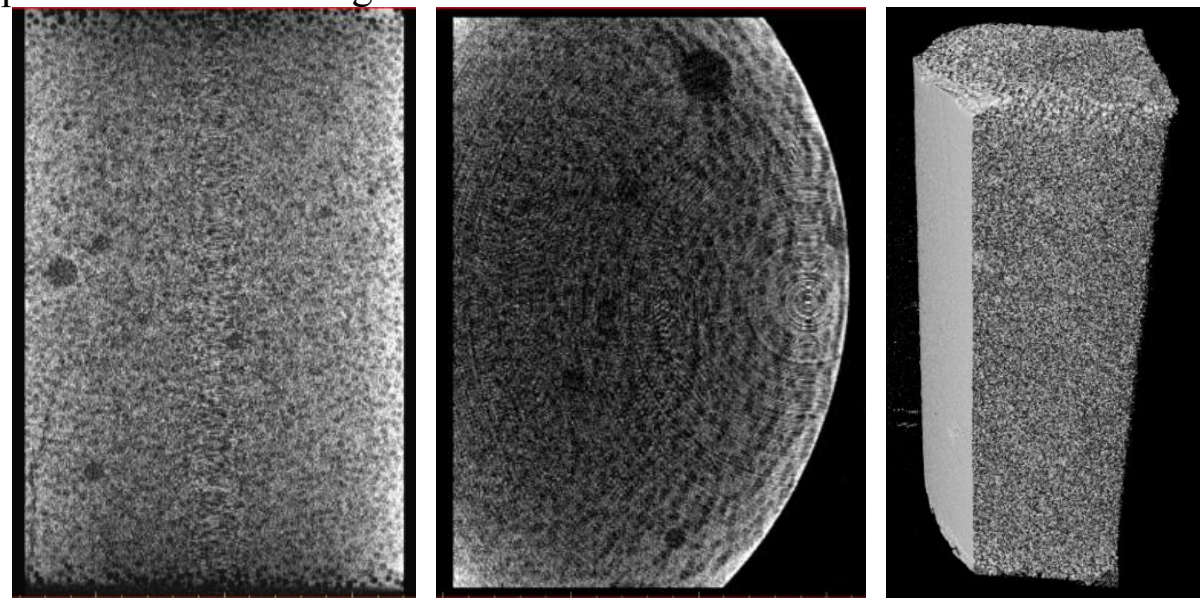

Figure 2. Resin particle distribution of sample 1 by CT scan $(50 \mu \mathrm{m}$ in accuracy).

As the scan results show, the distribution of resin particles in cement is relatively uniform. To further enhance precision and gain a clearer sight of resin particle distribution, sample 15 is cut into a $20 \mathrm{~mm}$ cube for microscopic CT scan, up to $10 \mu \mathrm{m}$ in accuracy, and overall distribution of resin particles in the sample is obtained as shown in Figure. 3, where black indicates resin particles, and the Figure on the right is the 3D model reconstructed with resin particles removed. The Figures show that resin particles are nearly spherical in relatively uniform particle size and randomly distributed. 

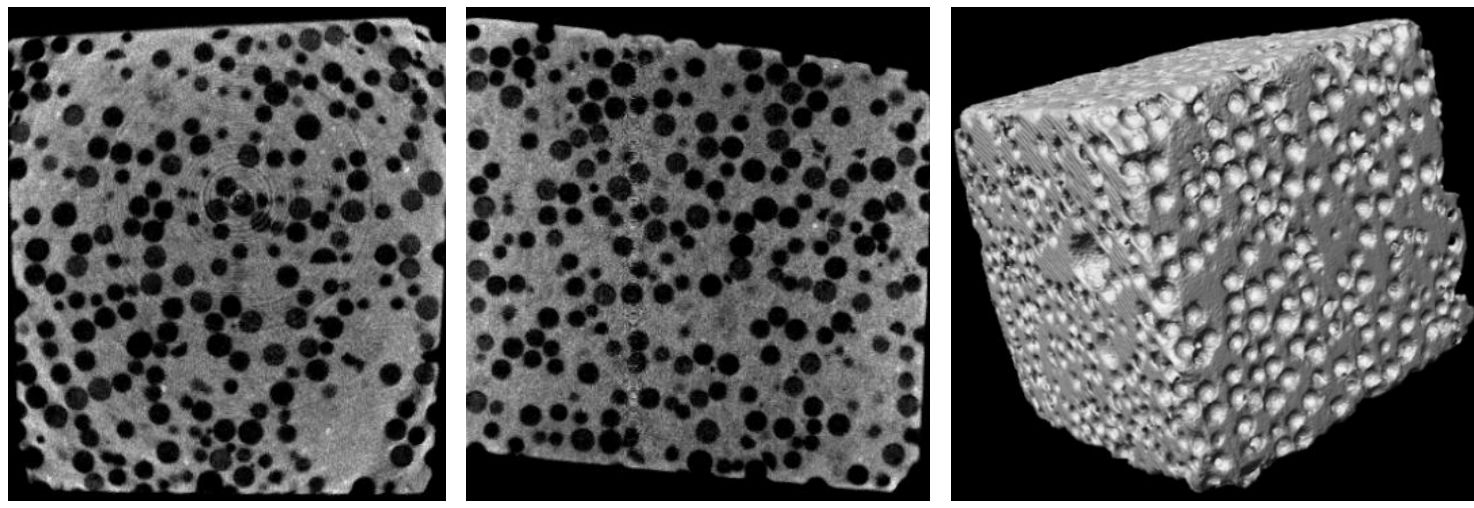

Figure 3. Resin particle distribution of sample 15 by CT scan (10 $\mu \mathrm{m}$ in accuracy).

\section{Compression test}

Compression test is performed by WAW-600C testing machine, with maximum pressure $600 \mathrm{kN}$, loading speed $20 \mathrm{KN} / \mathrm{min}$, ambient temperature $26{ }^{\circ} \mathrm{C}$, and humidity $53 \%$. During the compression test, sample 6, due to large defects appearing on the back, was discarded from the test. The stressstrain curve of the tested cement-solidified samples 1-7 is shown in Figure 4.

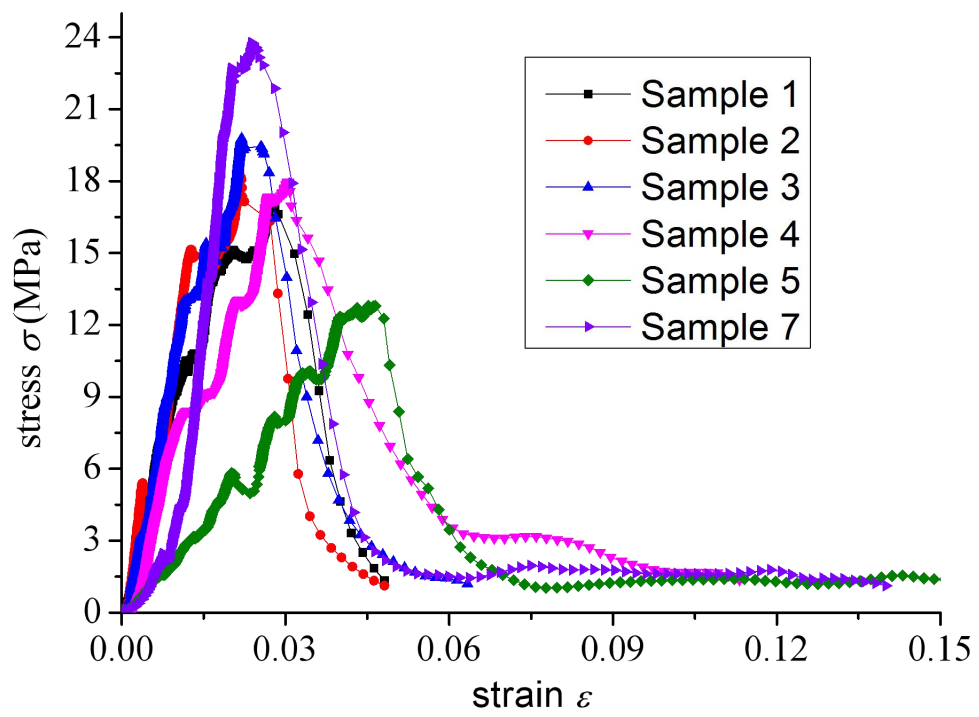

Figure 4. Stress-strain curve of samples 1-7.

As per GB 14569.1-2011, at least six parallel cement-solidified samples should be measured in the compression test to obtain average compressive strength as the test result. Remove the values exceeding plus or minus $20 \%$ of the average value of the six, and the average of the remaining five is the test result. If another one of the five still exceeds plus or minus $20 \%$ of the average of the five, then the test result of this group should be deemed invalid. The test result of cement-solidified samples 1-7 are shown in Table 1.

Table 1. Compression Test Results of Samples 1-7.

\begin{tabular}{ccccccc}
\hline Sample No. & 1 & 2 & 3 & 4 & 5 & 7 \\
\hline Maximum & & & & & 12.74 & \\
Compressive & 17.10 & 18.07 & 19.74 & 17.87 & $\begin{array}{c}\text { (Remove } \\
\text { d) }\end{array}$ & 23.60 \\
Strength (MPa) & & & & & (M) \\
\hline
\end{tabular}

Average

19.28

Sample 3 is taken as an example to observe the damage in compression test. Figure 5 shows that the sample starts flaking from around its body and completely collapses in the end, demonstrating a typical failure mode of brittle material under compression. 

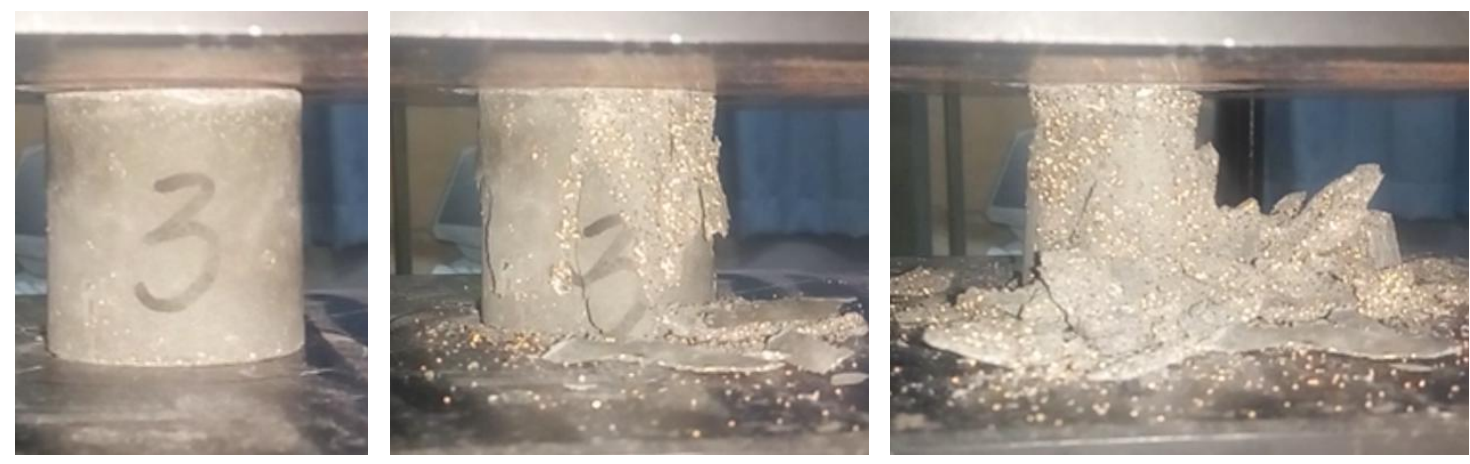

Figure 5. Compression failure of sample in compression test.

After soaking, samples 8-14 are polished on both the upper and lower sides to keep parallel. Average density is $1.81 \mathrm{~g} / \mathrm{cm} 3$. After soaking, the cement-solidified waste increases in density and becomes absorbent to some degree. Sample 11, due to obvious defects, was discarded from the loading test. The stress-strain curve of cement-solidified samples (samples 8-14) is shown in Figure 6.

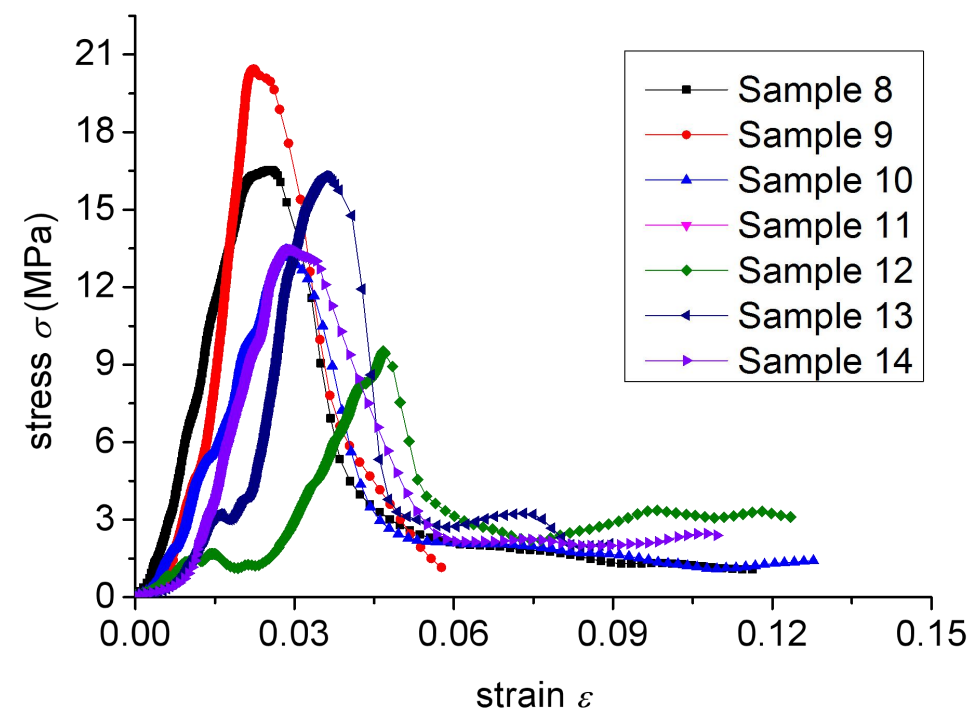

Figure 6. Stress-strain curve of cement-solidified samples after soaking.

The compression test results of cement-solidified samples after soaking are shown in Table 2 .

Table 2. Compression Test Results of Cement-solidified Samples after Soaking.

\begin{tabular}{ccccccc}
\hline Sample No. & 8 & 9 & 10 & 12 & 13 & 14 \\
\hline $\begin{array}{c}\text { Maximum } \\
\text { Compressive } \\
\text { Strength (MPa) }\end{array}$ & 16.53 & 20.40 & 113.42 & $\begin{array}{c}9.47 \\
\text { (Removed) }\end{array}$ & 16.29 & 13.43 \\
\hline Average & & \multicolumn{7}{c}{16.01} \\
\hline
\end{tabular}

Sample 9 is taken as an example to observe the damage in the compression test after soaking. From Figure. 7, we can see the sample, similar to samples without soaking, demonstrates a typical failure mode of brittle material under compression.

Figure. 4 and Figure. 6 show that cement solidified waste has a peak compressive strength, averaged 19.28 MPa before soaking and 16.01 MPa after soaking, indicating a slight decrease in maximum compressive strength but both still higher than the maximum compressive strength 7 $\mathrm{MPa}^{[5]}$ as stipulated in national standards. This means the maximum compressive strength of the samples meets national standards. After soaking, the samples absorb some water, increasing in density and decreasing in maximum compressive strength. 

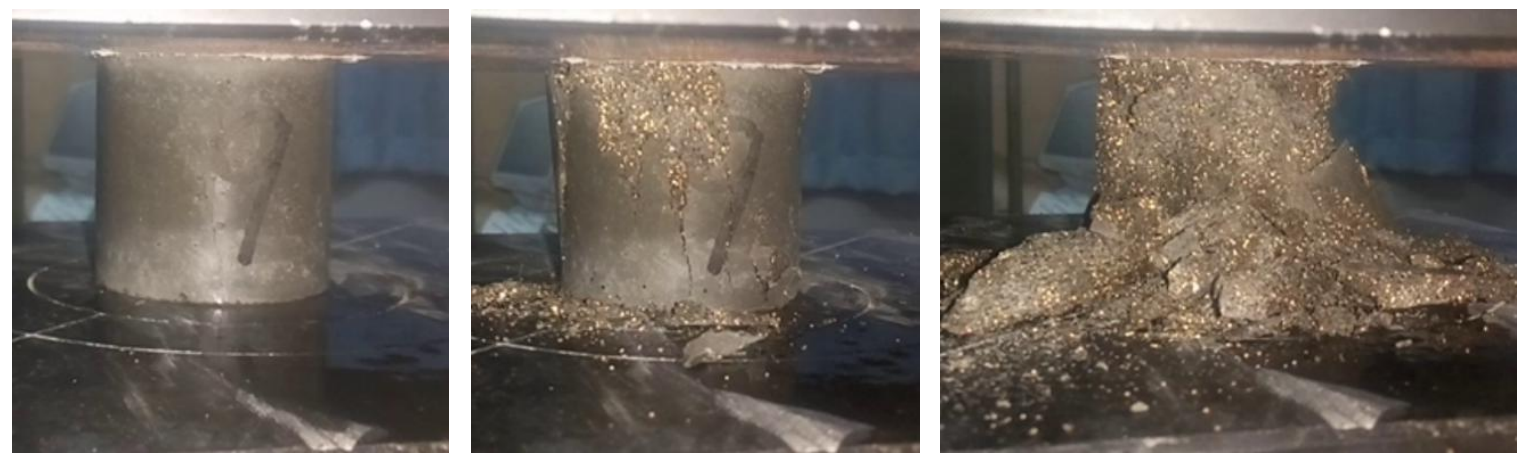

Figure 7. Compression failure of sample in compression test after soaking.

\section{Conclusion}

Based on density test, microscopic CT scan, and compression test on cement-solidified waste resin concentrate before and after soaking in deionized water, the following conclusions can be made: resin particles in cement-solidified waste are evenly distributed. After soaking, cement-solidified waste decreases in density and absorbs some water, resulting in a slight decrease in compressive strength. However, average compressive strength still meets GB 14569.1-2011 standards. Therefore, the solidification of waste resin concentrate with cement is feasible and effective, and could be used as a practical way of treating low and medium radioactive wastes.

\section{References}

[1] Uggla Y 2004 Risk and safety analysis in long-term perspective $J$. Futures 36: 549-64

[2] El-Ghonemy H, Watts L and Fowler L 2005. Treatment of uncertainty and developing conceptual models for environmental risk assessment $\mathrm{s}$ and radioactive waste disposal safety cases J. Environment International 31(1): 89-97

[3] Broden K and Olsson G 2003 Final disposal possibilities of radioactive waste components from ITER J. Fusion Engineering and Design 69(4): 695-697

[4] Micheline M, Kamali S and Guillon E 2004 Physicochemical equilibria of cement-based materials in aggressive environments experiment and modeling $J$. Cement Concrete Research 34(9): 1569-78

[5] Okada K, Nur R M and Fujii Y1999 The formation of explosive compounds in bitumen/nitrate mixtures J. Hazardous Mater 69(3): 245

[6] Nedoma J 1997 On a coupled Stefan-like problem in thermos-visco-plastic theology $J$. Computational Applied Mathematics 84(1): 45-80

[7] Roth G and Weisenburger S 2000 Vitrification of high level liquid waste: glass chemistry process chemistry and process technology J. Nuclear Engineering and Design 202 (2): 197 207

[8] Ewing R C 1999 Nuclear waste forms for actinides J. National Academy of Sciences Colloquium 3: 3432-39 